\title{
Lernen in entwicklungspolitischen Freiwilligendiensten - Eine lerntheoretische Reflexion
}

\author{
Dr. Susanne Krogull \\ Stellvertretende Leiterin des Jugendamtes der Erzdiözese Bamberg \\ susanne.krogull@eja-bamberg.de
}

\section{Zusammenfassung}

Der entwicklungspolitische Freiwilligendienst Weltwärts wird als Lerndienst definiert. Doch führt interkultureller Kontakt automatisch zu einem interkulturellen Lernerfolg und der Einsatz in einem Land der Entwicklungszusammenarbeit zu weltgesellschaftlichem und Globalem Lernen? Auf Basis theoretischer Reflexionen und konzeptioneller Erläuterungen werden Chancen und Risiken für Lernen in entwicklungspolitischen Freiwilligendiensten diskutiert. Zu den Chancen gehören die Erfahrbarkeit abstrakter Themen aufgrund einer Veränderung des persönlichen Nahbereichs über einen längeren Zeitraum, der Perspektivwechsel durch das Erleben anderer Lebenssituationen sowie ein Beitrag zur hybriden Identitätsentwicklung durch die aufgrund der Begegnung angeregte Selbstreflektion. Risiken sind, dass keine Abstraktion des Erlebten stattfindet und so persönliche Betroffenheit, jedoch kein Verständnis globaler Zusammenhänge entsteht sowie die Reproduktion paternalistischer Blickwinkel und kolonialer Verhältnisse.

Schlagwörter: Globales Lernen; Reflektion; interkulturelles Lernen; Postkolonialismus

\begin{abstract}
The development volunteer service Weltwärts is defined as a learning service. But does intercultural contact automatically lead to successful intercultural learning and international volunteering to global learning? Based on theoretical reflections and conceptual explanations opportunities and risks for learning in development volunteering are discussed. Opportunities are that by changing the personal social proximity and one's perspective over a longer period of time, abstract topics and life situations of others can be experienced. It may contribute to the hybrid identity development through the self-reflection of one's own way of life, values, and norms. Risks are that a lack of abstraction and reflection of the experience may lead to personal concern, but not to an understanding of global relationships and the reproduction of paternalistic views and colonial relationships.
\end{abstract}

Keywords: Global Learning; reflection; intercultural learning; postcolonialism

Mit dem vom Bundesministerium für wirtschaftliche Zusammenarbeit und Entwicklung (BMZ) eingeführten Weltwärts-Programm wurde mehr noch als in den Jahrzehnten davor für junge Menschen die Möglichkeit geschaffen, einen Freiwilligendienst in einem Land der Entwicklungszusammenarbeit zu leisten. Derzeit werden im Kontext des Weltwärts-Programms rund 7.000 Einsatzstellen von 
circa 180 Organisationen angeboten (Weltwärts o. J.a). Das Selbstverständnis des Programms hat sich in den zehn Jahren seiner Existenz weiterentwickelt und wird derzeit als ein entwicklungspolitischer Lerndienst beschrieben: „Weltwärts ist ein Lerndienst. Der Nord-Süd-Austausch und das gemeinsame interkulturelle Lernen stehen im Mittelpunkt. Die Freiwilligen sammeln Auslandserfahrungen und erwerben Sprachkenntnisse sowie persönliche Kompetenzen“ (Weltwärts o. J.b). Als Ziel wird darüber hinaus explizit die Förderung des Globalen Lernens genannt (Weltwärts o. J.c).

Angesichts dieses Selbstverständnisses wird mit diesem Artikel das Lernen in entwicklungspolitischen Freiwilligendiensten aus lerntheoretischer Perspektive reflektiert. Dazu werden zunächst das Konzept Globales Lernen erläutert und daran anschließend verschiedene Aspekte von Lernen in entwicklungspolitischen Freiwilligendiensten reflektiert. Es handelt sich hierbei um eine theoretische Reflexion, nicht um einen empirischen Nachweis von Lernen in Freiwilligendiensten. Es geht auch nicht um eine Kritik an der bisherigen Praxis, sondern der Artikel soll als Anregung verstanden werden, die bisherige Praxis (vielleicht aus neuen Perspektiven) zu reflektieren.

\section{Globales Lernen}

Weltwärts hat sich zum Ziel gesetzt, Globales Lernen zu fördern (Weltwärts o. J.c). Wenn von Globalem Lernen in entwicklungspolitischen Freiwilligendiensten gesprochen wird, kann der Eindruck entstehen, dass aufgrund der Verortung im globalen Kontext automatisch Globales Lernen stattfindet. Globales Lernen ist jedoch unabhängig davon zu sehen, wo gelernt wird, also ob in einem globalen, regionalen oder nationalen Kontext. Stattdessen beschreibt Globales Lernen ein pädagogisches Konzept. Dieses Konzept hat sich bereits in den 1990er Jahren konzeptionell in zwei verschiedene Richtungen entwickelt, die in der Praxis häufig parallel erscheinen, nämlich zum einen mit einer handlungstheoretischen Perspektive, zum anderen mit einer evolutionären, systemtheoretischen Perspektive (Scheunpflug 2007). Der Unterschied liegt vor allem in der Beschreibung der Ziele von Globalem Lernen. Während Globales Lernen aus handlungstheoretischer Perspektive auf die Vermittlung von Werten und normativen Inhalten fokussiert, die durch entsprechende Inhalte der Bildungsangebote erreicht werden sollen (z. B. Toleranz oder solidarisches Handeln) (im Überblick VENRO 2000), geht es bei Globalem Lernen aus systemtheoretisch oder evolutionär geprägter Perspektive vor allem um die Vermittlung von Kompetenzen, um mit der Komplexität des Lebens in der Weltgesellschaft und einer ungewissen Zukunft umzugehen (Scheunpflug/Schröck 2002). Diese unterschiedlichen Zielsetzungen scheinen sich zunächst nicht auszuschließen, allerdings gründen sie in unterschiedlichen lerntheoretischen Prämissen. Aus lerntheoretischer Sicht 
wird im handlungstheoretischen Ansatz davon ausgegangen, dass ein methodisches Vorgehen gepaart mit entsprechenden Inhalten zu einem anvisierten Ziel führt, dass also Werte und Wissen methodisch vermittelt werden können. Der systemtheoretische oder evolutionär geprägte Ansatz basiert auf der lerntheoretischen Prämisse, dass Lernen immer durch die Lernenden selbst gesteuert ist und Pädagogik keinen Zugriff darauf hat, was und wie die Lernenden lernen. Lernangebote, also methodisch vorbereitete Inhalte, können Lernen zwar anregen, sie können aber nicht das Ergebnis beschreiben, da dies von den Lernenden selbst gesteuert wird. Je nach dem zugrundeliegenden Verständnis von Globalem Lernen bzw. den lerntheoretischen Grundlagen wird man also vorsichtig sein mit der Beschreibung von Lernzielen im Sinne von Einstellungs- und Verhaltensänderungen.

In der Praxis (und nicht nur im Kontext von entwicklungspolitischen Freiwilligendiensten) werden diese lerntheoretischen Unterscheidungen oder Grundlegungen häufig nicht reflektiert.

\section{Lernen in Freiwilligendiensten}

Wenn von Lernen in Freiwilligendiensten gesprochen wird, geraten zunächst die Freiwilligen selbst als Zielgruppe in den Blick, für die der Freiwilligendienst angeboten wird. Diese lernen im Kontext des Programms sowohl formell in angebotenen Kursen (z. B. in Sprachkursen oder Vorbereitungsseminaren) als auch informell, also beiläufig im Alltag während des Aufenthalts in ihrem Einsatzland. Dieses Lernen geschieht sowohl individuell als auch in der Gruppe mit anderen Freiwilligen (anderen Ausländer*innen), im Kontext der Einsatzstelle (mit anderen Freiwilligen und den lokalen Projektpartner*innen) sowie in der Freizeit (eventuell auch wieder mit anderen Freiwilligen und/oder der lokalen Bevölkerung). Hier zeigt sich bereits, dass Lernen in entwicklungspolitischen Freiwilligendiensten im Kontext eines spezifischen sozialen Umfelds stattfindet. Dieses soziale Umfeld ist vor allem dadurch geprägt, dass es sich von dem bisherigen sozialen Umfeld der Freiwilligen unterscheidet. Da Lernen immer anknüpft an bereits gemachte Erfahrungen und vorhandenem Wissen, ist Lernen in entwicklungspolitischen Freiwilligendiensten immer ein Lernen in Auseinandersetzung mit Differenz. Dieses Lernen bezieht sich auf Wissen, aber auch auf Werte und Normen oder Einstellungen, die sich von denen der Freiwilligen unterscheiden. Hinzu kommen Aspekte wie Selbstwirksamkeit und verschiedene Kompetenzen, die im Rahmen eines solchen Freiwilligendienstes entwickelt werden können, zum Beispiel interkulturelle Kompetenz.

Doch führt der interkulturelle Kontakt automatisch zu einem interkulturellen Lernerfolg? Und: Führt der Einsatz in einem Land der Entwicklungszusammenarbeit automatisch zu weltgesellschaftlichem oder Globalem Lernen? Studien haben 
gezeigt, dass die von Allport (1954) entwickelte Kontakthypothese oder die sogenannte Immersions-Hypothese (Vande Berg/Paige u. a.. 2012), nach denen häufiger Kontakt zu Mitgliedern anderer Gruppen oder das Eintauchen in eine andere Kultur schon ausreichen, um interkulturell kompetent zu werden und Vorurteile abzubauen, so nicht länger haltbar sind, sondern dass es darum geht, wie dieser Kontakt gestaltet wird (Krogull 2018; Paige/Vande Berg 2010; Asbrand 2007; Thomas 1991). Es geht somit um die Frage, wie Lernprozesse gestaltet werden, damit die gewünschten Ziele erreicht werden.

Im Folgenden werden verschiedene Aspekte zu Lernen in entwicklungspolitischen Freiwilligendiensten beleuchtet. Manche dieser Aspekte zeigen Chancen auf, andere weisen auch auf Risiken hin. Dieser Artikel kann keine umfassende theoretische Reflexion bieten, greift jedoch einzelne Punkte heraus, die gerade für die Praxis von Bedeutung sein können.

\section{Veränderung des persönlichen Nahbereichs auf Zeit}

Entwicklungspolitische Freiwilligendienste bieten viele Möglichkeiten zum Lernen, da sie aufgrund ihrer Rahmenbedingungen und programmatischen Ausrichtung ein anderes Lernen als zum Beispiel in der Schule oder dem gewohnten kulturellen Umfeld ermöglichen. So bieten sie eine Veränderung des persönlichen Nahbereichs auf Zeit. Der Mensch hat sich in seiner Entwicklungsgeschichte auf den Nahbereich spezialisiert. Durch die Anpassung an das unmittelbare Umfeld mit seinen Problemen und Gefahren hat sich das Problemlöseverhalten der Menschen auf diesen Bereich fokussiert. Das, was sinnlich erfahrbar ist, was in dem direkten Umfeld geschieht, ist von Bedeutung (Scheunpflug 2001). Dadurch sind Themen, die das eigene Leben direkt betreffen leichter thematisierbar als abstrakte Themen wie fairer Handel oder der Einsatz von Pestiziden auf Baumwolfarmen in Afrika, die nicht zum direkten Erfahrungsraum der Lernenden gehören. Durch den Einsatz in Ländern der Entwicklungszusammenarbeit ermöglichen entwicklungspolitische Freiwilligendienste die Auseinandersetzung mit eben solchen Themen, die zu Hause abstrakt, da nicht Teil des eigenen Nahbereichs sind, jedoch zum Lebensalltag und dem sozialen Nahbereich im Einsatzland gehören. Abstrakte Themen des Heimatlandes dringen durch die räumliche Veränderung in den persönlichen Nahbereich ein. Die Auswirkungen der Globalisierung auf den Globalen Süden werden erfahrbar, Armut wird erlebbar und dadurch entsteht die Chance einer reflektierten Auseinandersetzung aufgrund von persönlichen Erfahrungen.

\section{Perspektivenwechsel}

Durch den direkten Kontakt und das Zusammenleben mit Menschen in Ländern der Entwicklungszusammenarbeit und durch das Wahrnehmen von 
Perspektiven sowie das Erleben von Lebenssituationen, die sich von dem gewohnten Leben der Freiwilligen zu Hause unterscheiden, eröffnen entwicklungspolitische Freiwilligendienste Differenzerfahrungen in alltäglichen Kontexten. Während im Heimatland das Einüben von Perspektivenwechsel oftmals nur theoretisch möglich ist, wird im Rahmen des entwicklungspolitischen Freiwilligendienstes durch die direkte Differenzerfahrung und die Möglichkeit, diese Erfahrungen vor Ort und mit den Beteiligten zu diskutieren und zu reflektieren, ein Perspektivenwechsel ermöglicht, der nicht nur auf einer abstrakten Rolleneinnahme beruht, sondern auf den Erfahrungen der Freiwilligen selbst. So kann ein Perspektivenwechsel ermöglicht werden, der zu einem besseren und detaillierteren Verständnis von Situationen, Haltungen, Werten und Verhaltensmustern führen kann, gerade weil die andere Perspektive „live“ vor Ort erfahrbar und reflektierbar wird.

\section{Identitätsbildung durch Selbstreflexion}

Der entwicklungspolitische Freiwilligendienst ermöglicht nicht nur Perspektivenwechsel, sondern bietet auch Möglichkeiten erhöhter Selbstreflexion. Vor dem Hintergrund der gemachten Differenzerfahrungen können die eigene Lebensweise, die eigenen Werte und Normen, eigene Einstellungen, Handlungsweisen und -muster reflektiert werden. Dabei liegt der Fokus an dieser Stelle nicht auf einem Perspektivenwechsel zum besseren Verständnis fremder Perspektiven, sondern einer bewussten Auseinandersetzung mit Dingen, die im heimischen Kontext als selbstverständlich angesehen oder aufgrund ihrer „Normalität“ unreflektiert bleiben. Entwicklungspolitische Freiwilligendienste können durch diese Selbstreflexion zur Identitätsentwicklung der jungen Freiwilligen beitragen. Der entwicklungspolitische Freiwilligendienst kann aufgrund der gemachten Lernerfahrungen eine prägende Funktion in der hybriden Identitätsentwicklung junger Menschen haben.

\section{Abstraktionsfähigkeit statt Erfahrungswissen}

Lernen bedeutet, von einer persönlichen Erfahrung abstrahieren zu können, um dadurch die dahinterliegenden Strukturen und Mechanismen zu erkennen. Lernen braucht somit Abstraktion. Wenn persönliche Erfahrungen reflektiert werden, können durch die Reflexion und die Auseinandersetzung mit den persönlichen Erfahrungen Bezüge hergestellt und Zusammenhänge erkannt werden. Die Erfahrung an sich wird somit erst zum Ausgangspunkt des Lernprozesses. Findet keine Abstraktion des erworbenen Erfahrungswissens statt, besteht die Gefahr, dass zum Beispiel die Armutserfahrung auf der Ebene der persönlichen Betroffenheit bleibt, jedoch nicht zu einem Verständnis oder einem Erkennen von globalen Zusammenhängen führt. Beim Lernen in entwicklungspolitischen 
Freiwilligendiensten geht es daher um die abstrakte Generalisierung von konkreten Erfahrungen.

\section{Begrenztheit des eigenen Wissens}

Damit die abstrakte Generalisierung von konkreten Erfahrungen nicht zur Ausbildung von Stereotypen führt, geht es gleichzeitig darum, das eigene Wissen als begrenzt anzuerkennen. Nur weil jemand einen entwicklungspolitischen Freiwilligendienst in einer landwirtschaftlichen Einrichtung in Uganda gemacht hat, werden diese (ehemaligen) Freiwilligen dadurch nicht automatisch zu Expert*innen für Landwirtschaft in Uganda, für Uganda und die ugandische Lebensweise allgemein und generell für Afrika. Wissen ist begrenz und entwickelt sich weiter und es muss deutlich werden, dass auch durch einen entwicklungspolitischen Freiwilligendienst von zwölf Monaten nicht ein ganzes Land verstanden werden kann, sondern dass das erworbene Erfahrungswissen auf einen Ausschnitt begrenzt ist. Freiwillige werden nach ihrer Rückkehr häufig als „Expert*innen“ für ein Land oder sogar den ganzen Kontinent angesehen. Es bedarf daher einer Reflexion, die dieses vermeintliche Expertentum und die Begrenztheit des eigenen Wissens thematisiert. Es gilt das Lebens als komplex wahrzunehmen und im Rahmen von entwicklungspolitischen Freiwilligendiensten die Kompetenz zu erlangen, mit dieser Komplexität umgehen zu lernen.

\section{Begegnung auf Augenhöhe, statt Reproduktion paternalistischer Blickweisen und kolonialer Verhältnisse}

Ein Aspekt, der nicht nur für das Lernen in entwicklungspolitischen Freiwilligendiensten wichtig ist, sondern der eng mit dem Selbstverständnis bzw. dem gesellschaftlichen Verständnis solcher Programme verknüpft ist, ist der häufig genannte Begriff „Begegnung auf Augenhöhe“ als Zielperspektive für entwicklungspolitische Freiwilligendienste. Mit diesem Begriff ist die Programmatik und Zielperspektive verbunden, dass Freiwillige auf einer Ebene mit den Menschen in den Einsatzstellen stehen, dass es um ein Miteinander geht, nicht um ein „für jemanden“. Mit dieser Zielperspektive wird zum Beispiel Weltwärts explizit als ein „Lerndienst“ beschrieben. Junge Menschen sollen als Lernende an diesem Programm teilnehmen, nicht als Expert*innen. Dieser Anspruch wird allerdings oftmals durch die Einsatzstellen konterkariert, in denen die Freiwilligen Tätigkeiten durchführen, für die Menschen aus dem Einsatzland eine Ausbildung bräuchten sowie durch das Verständnis von Entwicklungspolitik und Weltwärts als einem Instrument der Entwicklungszusammenarbeit. Auch wenn sich das Verständnis von Entwicklungszusammenarbeit (das sich bereits in dem Wort Entwicklungszusammenarbeit statt Entwicklungshilfe niederschlägt) in entwicklungspolitischen Kreisen geändert hat, so kann davon ausgegangen werden, 
dass dieses Verständnis noch nicht alle Bereiche der Gesellschaft durchdrungen hat und im allgemeinen Sprachgebrauch immer noch häufig von Entwicklungshilfe oder dem Entwicklungshilfeministerium, statt dem Bundesministerium für wirtschaftliche Zusammenarbeit und Entwicklung gesprochen wird. Doch was hat dieses Begriffsverständnis mit Lernen in entwicklungspolitischen Freiwilligendiensten zu tun und warum erscheint es wichtig, dies zu thematisieren? Das Selbst- bzw. Fremdverständnis setzt den Rahmen für das Lernen in den entwicklungspolitischen Freiwilligendiensten. Wenn jemand einen entwicklungspolitischen Freiwilligendienst macht mit dem Verständnis, durch die eigene Hilfe zur Entwicklung eines Landes beizutragen, dann wird sich der Lernprozess anders gestalten als bei jemanden, der in ein Land fährt, um dort etwas zu lernen. Es ist also zum einen ratsam bei der Auswahl der Einsatzstellen sowie bei der Begleitung darauf zu achten, dass dieser Aspekt immer wieder thematisiert wird. Hier kommt vor allem den pädagogischen (Begleit-)Seminaren eine wichtige Rolle zu, da in ihnen nicht nur die Alltagsprobleme der Freiwilligen thematisiert werden können, sondern vor allem auch die Reflexion der Position der Freiwilligen als Deutsche/Europäer*innen und der gesellschaftlich politischen Verhältnisse. Andernfalls besteht das Risiko, dass entwicklungspolitische Freiwilligendienste koloniale Strukturen re-etablieren und es aufgrund eines Überlegenheitsgefühls der Freiwilligen zu paternalistischen und rassistischen Einstellungen kommt (Krogull 2018).

\section{Engagement nach dem Freiwilligendienst}

Was gelernt wurde zeigt sich nicht nur auf der Ebene von Wissen, Einstellungen und Kompetenzen, sondern auch auf der Handlungsebene der Freiwilligen. Das Engagement der Freiwilligen nach ihrer Rückkehr kann daher als ein Produkt ihres Lernprozesses gesehen werden. Es gibt unterschiedliche Aktionsformen nach dem Freiwilligendienst, die teilweise von Entsendeorganisationen unterstützt und angeregt, teilweise von ehemaligen Freiwilligen selbst initiiert werden und die von Vereinsgründungen über politische Aktionen bis hin zu Bildungsveranstaltungen reichen. Mit Blick auf Lernen in entwicklungspolitischen Freiwilligendiensten muss sich die Frage gestellt werden, welche Aktionsformen adäquat mit den Programmzielen bzw. den Zielen des Globalen Lernens einhergehen. Vereinsgründungen oder Projekte mit dem Zweck, Spenden und finanzielle Unterstützung für Projekte in den ehemaligen Einsatzländern der Freiwilligen zu generieren, laufen Gefahr, gerade nicht eine Begegnung auf Augenhöhe und ein Verständnis von globalen Strukturen zu fördern, sondern stattdessen das Bild vom entwicklungsbedürftigen, armen Globalen Süden, der auf finanzielle Unterstützung angewiesen ist, zu festigen. Im Sinne des handlungstheoretischen Ansatzes von Globalem Lernen können solche Aktivitäten als tätige Solidarität oder als Zeichen für Empathie gewertet und somit positiv konnotiert 
werden. Aus der Perspektive des systemtheoretischen oder evolutionären Ansatzes des Globalen Lernens jedoch können solche Aktivitäten - wenn sie alleine stehen und nicht gleichzeitig mit einem Engagement zum Beispiel für die Veränderung von (politischen) Strukturen einhergehen - als eine Aktivität gesehen werden, die die Komplexität der Welt nicht adäquat abbildet und das Verständnis für komplexe globale Zusammenhänge als unzureichend beurteilt werden kann. Aus systemtheoretischer Perspektive wäre ein Engagement im fairen Handel oder politische Aktivitäten positiver zu bewerten als die Organisation finanzieller Mittel, da sie als Zeichen dafür gesehen werden können, dass globale Zusammenhänge verstanden und Bezüge zwischen dem eigenen Verhalten im Globalen Norden und den Lebensbedingungen im Globalen Süden hergestellt werden.

An dieser Stelle geht es nicht darum, eine Aktivitätsform als besser oder schlechter darzustellen. Stattdessen soll vor dem Hintergrund unterschiedlicher lerntheoretischer Prämissen gezeigt werden, dass ein und dieselbe Aktivität je nach lerntheoretischer Perspektive unterschiedlich bewertet werden kann.

\section{Zusammenfassung}

Weltwärts als entwicklungspolitischer Freiwilligendienst wird auf programmatischer Ebene als Lerndienst bezeichnet. Um dies auch in der Praxis umzusetzen erscheint es hilfreich, lerntheoretische Perspektiven zu berücksichtigen. Der vorliegende Artikel liefert dazu einige Anregungen. Grundsätzlich gilt zu beachten, dass Lernen aus lerntheoretischer Perspektive als ein eigenständiger Prozess des Lernenden selbst verstanden wird. Methodische und inhaltliche Einheiten können vor dieser Prämisse immer nur Lernangebote sein. Inwiefern diese genutzt werden, hängt von den Lernenden selbst ab. Lernen als ein von den Lernenden selbst gesteuerter Prozess spielt auch bei dem systemtheoretisch oder evolutionär geprägten Verständnis von Globalem Lernen eine Rolle, indem es vorrangig auf Kompetenzen für ein Leben in der Weltgesellschaft abzielt und nicht auf eine Veränderung von Werten und Haltungen, welche dem handlungstheoretisch geprägten Verständnis von Globalem Lernen zugrunde liegt. Wird in den Programmen der entwicklungspolitischen Freiwilligendienste Bezug genommen zu Globalem Lernen, dann erscheint es notwendig, sich zu den unterschiedlichen Ausrichtungen Globalen Lernens (und damit einhergehend zu einem bestimmten Lernverständnis) zu verhalten, damit Lernziele lerntheoretisch fundiert beschrieben werden können bzw. Lerntheorien nicht widersprechen. Dabei bieten entwicklungspolitische Freiwilligendienste das Potenzial, beide Verständnisse von Globalem Lernen miteinander zu verschmelzen und sowohl dem systemtheoretischen Verständnis zu entsprechen, ohne dabei die handlungstheoretische Perspektive aufgeben zu müssen. Dazu bedarf es aber einer theoretisch fundierten 
Auseinandersetzung mit Lernen im Allgemeinen und in entwicklungspolitischen Freiwilligendiensten im Speziellen.

Bei der lerntheoretischen Reflexion wurde immer wieder auf die Notwendigkeit von Abstraktion und Reflexion hingewiesen. Daher kommt sowohl den pädagogischen (Begleit-)Seminaren, als auch den Mentor*innen vor Ort eine besondere Bedeutung zu. Sie ermöglichen einen Rahmen, in dem Erfahrungen reflektiert und in einen größeren Kontext gestellt werden und dadurch zum Ausgangspunkt von Lernprozessen werden können. So kann der Gefahr vorgebeugt werden, dass erworbenes erfahrungsbasiertes Wissen auf der Ebene der Erfahrung verbleibt, sich ein vermeintliches Expertentum oder gar Paternalismus oder Rassismus entwickeln. Gleichzeitig können durch sie Lernangebote gemacht werden, die zum Kompetenzerwerb der Freiwilligen beitragen können.

Auch wenn der Lernprozess durch die Freiwilligen selbst gesteuert wird, so geschieht er doch in Interaktion mit dem sie umgebenden Umfeld. Daher gilt grundsätzlich auch zu beachten, dass in entwicklungspolitischen Freiwilligendiensten nicht nur die Freiwilligen lernen, sondern durch die Interaktion auch das Umfeld, sei es im Einsatzland oder im Heimatland (auf diesen Aspekt konnte in diesem Artikel leider nicht näher eingegangen werden).

Die Planung von Lernen in entwicklungspolitischen Freiwilligendiensten bedarf einer pädagogischen Reflexion, denn Lernen geschieht nicht im luftleeren Raum. Um Lernen tatsächlich zu ermöglichen, ist es notwendig, sich mit lerntheoretischen Ansätzen auseinanderzusetzen, auf deren Grundlagen dann Lernen geplant/ angeregt werden kann bzw. Lernziele beschrieben werden können. Je nach Verständnis von Globalem Lernen können sich diese Ziele unterscheiden.

In der Praxis von entwicklungspolitischen Freiwilligendiensten (aber nicht nur dort) werden lerntheoretische Unterscheidungen oder Grundlegungen häufig nicht reflektiert. Pädagogische Begriffe und Konzepte werden zwar verwendet bzw. in den Programmen auf sie rekurriert, jedoch oftmals ohne die unterschiedlichen Perspektiven und theoretischen Grundlagen in den Blick zu nehmen. Die Berücksichtigung von lerntheoretischen Perspektiven und Grundlagen von Lernen kann jedoch dazu beitragen, dass (Lern-)Ziele und Wirkungsannahmen realistisch und fundiert beschrieben werden können. Ein paar dieser Perspektiven wurden in diesem Artikel benannt, die sowohl in Bezug zur Programmatik von Weltwärts stehen (Begegnung auf Augenhöhe, Globales Lernen, Perspektivenwechsel) als auch zu Lernen allgemein (Nahraumorientierung, Abstraktion, Selbstreflexion). Sie stellen lediglich eine Auswahl dar, ohne Anspruch auf Vollständigkeit. Sie können jedoch dabei helfen, den Anspruch eines entwicklungspolitischen Lerndienstes (lern-)theoretisch fundiert umzusetzen. 


\section{Literaturverzeichnis}

Allport, Gordon W. (1954): The nature of prejudice, Cambridge.

Asbrand, Barbara (2007): Partnerschaft - eine Lerngelegenheit?, in: Zeitschrift für internationale Bildungsforschung und Entwicklungspädagogik, Jg. 30, Heft 3, S. 8-12.

Illeris, Knud (2010): Lernen verstehen. Bedingungen erfolgreichen Lernens, Bad Heilbrunn.

Krogull, Susanne (2018): Weltgesellschaft verstehen. Eine internationale, rekonstruktive Studie zu Perspektiven junger Menschen, Wiesbaden.

Paige, Michael and Michael Vande Berg (2012): Why students are and are not learning abroad. A review of recent research, in: Student Learning Abroad: What Our Students Are Learning, What They're Not, and What We Can Do About It, edited by Michael Vande Berg, Michael Paige and Kris Hemming Lou, Sterling, S. 29-58.

Scheunpflug, Annette (2001): Biologische Grundlagen des Lernens. Ein Studienbuch, Berlin.

Scheunpflug, Annette (2007): Die konzeptionelle Weiterentwicklung des Globalen Lernens. Die Debatten der letzten zehn Jahre, in: Jahrbuch Globales Lernen 2007/2008. Standortbestimmungen, Praxisbeispiele, Perspektiven. Bonn, S. 11-21.

Scheunpflug, Annette und Nikolaus Schröck (2002): Globales Lernen, Stuttgart.

Thomas, Alexander (Hrsg.) (1991): Kulturstandards in der internationalen Begegnung, Saarbrücken-Fort Lauderdale.

Vande Berg, Michael, R. Michael Paige and Kris Hemming Lou (Hrsg.): Student Learning Abroad: What Our Students Are Learning What They're Not, and What We Can Do About It, Sterling.

VENRO - Verband Entwicklungspolitik und Humanitäre Hilfe (2000): „Globales Lernen“ als Aufgabe und Handlungsfeld entwicklungspolitischer Nichtregierungsorganisationen. Grundsätze, Probleme und Perspektiven der Bildungsarbeit des VENRO und seiner Mitgliedsorganisationen. VENRO Arbeitspapier 10, Bonn.

Weltwärts (o. J.): Historie. Ein staatliches Förderprogramm wird zum Erfolg, www.weltwaerts.de/de/historie.html (15.01.2019).

Weltwärts (o. J.b): Programm. Der entwicklungspolitische Freiwilligendienst stellt sich vor, www.weltwaerts.de/de/programm.html (15.01.2019).

Weltwärts (o. J.c): Ziele. Weltwärts ist ein entwicklungspolitischer Lerndienst, www.weltwaerts.de/de/ziele.html (15.01.2019). 